\title{
EXTREMAL SETS OF $p$-TH SECTIONAL CURVATURE
}

\author{
ANN STEHNEY
}

The object of this paper is to study the pointwise behavior of the $p$-th order sectional curvature function $\sigma$ of a Riemannian manifold $M$. At $m \in M, \sigma$ is a real-valued function on the compact Grassmann manifold $\mathscr{G}$ of $p$-planes in the tangent space $M_{m}$ of $M$ at $m$. We shall describe the subsets of $\mathscr{G}$ on which $\sigma$ assumes its maximum and minimum.

We shall work in the setting of an arbitrary inner product space $V$ and arbitrary integer $p(2 \leq p \leq \operatorname{dim} V)$. A $p$-th curvature operator is a selfadjoint linear transformation $R: \Lambda^{p} \rightarrow \Lambda^{p}$, where $\Lambda^{p}=\Lambda^{p}(V)$ has the inner product induced by that of $V$. (For example, if $V=M_{m}$ and $p$ is even, the Riemannian $p$-th curvature operator $R_{p}$ as defined by Thorpe [4].) The Grassmann manifold $\mathscr{G}$ of oriented $p$-planes in $V$ is viewed as a subset of the unit sphere in $\Lambda^{p}$.

For $R$ in the vector space $\mathscr{R}$ of $p$-th curvature operators, we consider its sectional curvature $\sigma_{R}: \mathscr{G} \rightarrow \boldsymbol{R}$ given by

$$
\sigma_{R}(\mathscr{P})=\langle R(\mathscr{P}), \mathscr{P}\rangle \quad(\mathscr{P} \in \mathscr{G}) .
$$

With respect to the inner product

$$
\langle T, U\rangle=\operatorname{tr} T \circ U \quad(T, U \in \mathscr{R}),
$$

$\mathscr{R}$ decomposes orthogonally into $\mathscr{S} \oplus \mathscr{B}$, where $\mathscr{S}$ is the span of the Grassmann quadratic $p$-relations which define $\mathscr{G}$ :

$$
\mathscr{G}=\left\{\alpha \in \Lambda^{p} \mid\|\alpha\|=1 \text { and }\langle S(\alpha), \alpha\rangle=0 \text { for all } S \in \mathscr{S}\right\},
$$

and $\mathscr{B}$ is the subspace of operators satisfying generalized Bianchi identities (for $p=2$, the usual first Bianchi identity for a Riemannian curvature operator). The first section of this paper is devoted to this decomposition of $\mathscr{R}$. We show that $R \in \mathscr{B}$ and $\sigma_{R} \equiv 0$ imply $R=0$. It follows that $\mathscr{S}$ is the set of curvature operators whose sectional curvature is identically zero.

In $\S 2$ we find a basis for $\mathscr{S}$. This gives a reduction of the Grassmann quadratic $p$-relations to a minimal set of conditions. We believe that this result in exterior algebra is new and so we state it here (cf. Lemma 1.1).

Received March 13, 1972. 
Theorem A. Let $\left\{x_{1}, \cdots, x_{n}\right\}$ be any basis for $V$. The p-vector

$$
\alpha=\sum_{i_{1}<\cdots<i_{p}} \alpha_{i_{1} \cdots i_{p}} x_{i_{1}} \wedge \cdots \wedge x_{i_{p}}
$$

is decomposable if and only if its Plücker coordinates $\alpha_{i_{1} \ldots i_{p}}$ satisfy

$$
\sum_{k=1}^{p+1}(-1)^{k} \alpha_{i_{1} \cdots \hat{i}_{k} \cdots i_{p+1}} \alpha_{i_{k} j_{1} \cdots j_{p-1}}=0
$$

for all $1 \leq i_{1}<\cdots<i_{p+1} \leq j_{p-1}, \quad 1 \leq j_{1}<\cdots<j_{p-1} \leq n$ such that $\left\{j_{1}, \cdots, j_{p-1}\right\} \not \subset\left\{i_{1}, \cdots, i_{p+1}\right\}$.

Our main theorem asserts that for any curvature operator $R$, the set of $p$-planes in $V$ on which $\sigma_{R}$ assumes its maximum or minimum is the intersection of $\mathscr{G}$ with a linear subspace of $\Lambda^{p}$. In particular, we prove

Theorem B. If $R \in \mathscr{R}$ and $\sigma_{R} \geq 0$, then there exists $S \in \mathscr{S}$ such that

$$
\left\{\mathscr{P} \in \mathscr{G} \mid \sigma_{R}(\mathscr{P})=0\right\}=\mathscr{G} \cap \operatorname{Ker}(R-S) .
$$

The case of an arbitrary minimum or maximum is an easy consequence of this special case.

To prove the main theorem, we shall prove that if $\mathscr{P}$ is a critical point of $\sigma_{R}$, and $\sigma_{R}(\mathscr{P})$ vanishes, then there exists $S_{\mathscr{g}} \in \mathscr{S}$, in fact unique modulo elements of $\mathscr{P}$ which annihilate $\mathscr{P}$, for which $\mathscr{P} \in \operatorname{Ker}\left(R-S_{\mathscr{g}}\right)$. Since $\Lambda^{p}$ is finite dimensional, we may piece together these unique $S_{\mathscr{F}}$ 's into an $S$ which has the desired property.

The theorem is a generalization of Thorpe's result [5] for $p=2$. We have omitted proofs of several steps where the obvious generalization of Thorpe's proof is valid. The main difficulty in the proof for $p>2$ is in finding an $S$ which "works" simultaneously for two arbitrary planes $\mathscr{P}$ and $Q$ on which $\sigma_{R}$ vanishes. Such an $S$ must have the correct orthogonal projections, namely $S_{\mathscr{g}}$ and $S_{Q}$, onto the orthogonal complements of the annihilators of $\mathscr{P}$ and $Q$, and so $S$ exists if and only if $S_{\mathscr{g}}$ and $S_{Q}$ have the same projections onto the intersection of these orthogonal complements. We use our basis for $\mathscr{S}$ to find this intersection explicitly. If $(\mathscr{P}+Q) /\|\mathscr{P}+Q\|$ is not in $\mathscr{G}$, this intersection has dimension 1 and we find a basis for it. If $(\mathscr{P}+Q) /\|\mathscr{P}+Q\|$ is in $\mathscr{G}$, then $\mathscr{P}$ and $Q$ determine a circle in $\mathscr{G}$ of planes on which $\sigma_{R}$ vanishes. The fact that zero is an extreme value of $\sigma_{R}$ is used only in this case and only to show that each plane in the circle is a critical point of $\sigma_{R}$. An example shows that the hypothesis of the theorem cannot be weakened to include the case of a nonextreme critical value of $\sigma_{R}$.

Although the theorem holds in a general setting, our main interest is the $p$-th curvature operator arising on a tangent space $M_{m}$ of a Riemannian manifold. Specifically, let $\tilde{R}$ denote the Riemannian curvature tensor at $m$ in $M$, and let $R\left(x_{1}, x_{2}, x_{3}, x_{4}\right)$ denote the real number $\left\langle\tilde{R}\left(x_{1}, x_{2}\right) x_{3}, x_{4}\right\rangle$ for $x_{i} \in M_{m}$. For even $p$, the Riemannian $p$-th curvature operator $R_{p}$ is defined in [4] by 


$$
\begin{aligned}
& \left\langle R_{p}\left(u_{1} \wedge \cdots \wedge u_{p}\right), v_{1} \wedge \cdots \wedge v_{p}\right\rangle \\
& \quad=\frac{1}{2^{p / 2} p !} \sum_{\alpha, \beta \in S_{p}} \varepsilon(\alpha) \varepsilon(\beta) R\left(u_{\alpha_{1}}, u_{\alpha_{2}}, v_{\beta_{1}}, v_{\beta_{2}}\right) \cdots R\left(u_{\alpha_{p-1}}, u_{\alpha_{p}}, v_{\beta_{p-1}}, v_{\beta}\right)
\end{aligned}
$$

where $u_{i}, v_{j} \in M_{m}, S_{p}$ is the permutation group on $(1, \cdots, p)$, and $\varepsilon(\alpha)$ is the sign of $\alpha \in S_{p}$. If $n(=\operatorname{dim} M)$ is even, the sectional curvature of $R_{n}$ is the Lipschitz-Killing curvature of $M$ at $m$ and therefore (the value at $m$ of) the Euler integrand according to the generalized Gauss-Bonnet theorem. This suggests that more geometric information about how the curvature tensor depends on sectional curvature might help to resolve the conjecture that a Riemannian manifold with positive sectional curvature $(p=2)$ has a positive Euler characteristic. Thorpe [6] has characterized the curvature tensors of positive sectional curvature for $n \leq 4$. If the generalization of his result proved valid, it would provide a geometric explanation for the phenomenon of our Theorem B.

This paper includes the main results of the author's doctoral dissertation at the State University of New York at Stony Brook. I take great pleasure in thanking Professor John A. Thorpe for his guidance and encouragement as both my teacher and my advisor.

\section{Decomposition of the space of curvature operators}

Let $V$ be an $n$-dimensional real inner product space. For $p$ an integer, $1 \leq p \leq n$, let $\Lambda^{p}(V)$ or simply $\Lambda^{p}$ denote the space of $p$-vectors of $V$. We recall several facts about $\Lambda^{p}$. A $p$-vector $\alpha$ is called decomposable if it can be written in the form $u_{1} \wedge \cdots \wedge u_{p}$ with $u_{i} \in V$. Any basis $E=\left\{e_{1}, \cdots, e_{n}\right\}$ for $V$ induces a basis $\left\{e_{i_{1}} \wedge \cdots \wedge e_{i_{p}} \mid i_{1}<\cdots<i_{p}\right\}$ for $\Lambda^{p}$ consisting of decomposable $p$-vectors. It follows that $\operatorname{dim} \Lambda^{p}=C_{n, p}$. An arbitrary $p$-vector is of the form

$$
\alpha=\sum \alpha_{i_{1} \cdots i_{p}} e_{i_{1}} \wedge \cdots \wedge e_{i_{p}},
$$

where the summation extends over all $1 \leq i_{1}<\cdots<i_{p} \leq n$. The coefficients $\alpha_{i_{1} \cdots i_{p}}$, skew symmetric in their indices, are called the Plücker coordinates of $\alpha$ with respect to the basis $E$. We define an inner product for $\Lambda^{p}$ on decomposable $p$-vectors by

$$
\left\langle u_{1} \wedge \cdots \wedge u_{p}, v_{1} \wedge \cdots \wedge v_{p}\right\rangle=\operatorname{det}\left[\left\langle u_{i}, v_{j}\right\rangle\right]
$$

where $u_{i}, v_{j} \in V$. It is easily seen that the basis for $\Lambda^{p}$ induced as above by an orthonormal basis for $V$ is itself orthonormal.

We may identity the Grassmann manifold $\mathscr{G}$ of oriented $p$-dimensional subspaces of $V$ with the submanifold of $\Lambda^{p}$ consisting of decomposable $p$-vectors of length one by 


$$
\mathscr{P} \leftrightarrow u_{1} \wedge \cdots \wedge u_{p},
$$

where $\left\{u_{1}, \cdots, u_{p}\right\}$ is an oriented orthonormal basis for $\mathscr{P}$. Elements of $\mathscr{G}$ will be called $p$-planes.

We will say that $\alpha \in \Lambda^{p}$ contains $x \in V$ as a wedge factor if $\alpha=x \wedge \eta$ for some $\eta \in \Lambda^{p-1}(V)$.

Let $\mathscr{R}^{p}$, or simply $\mathscr{R}$ if $p$ is fixed, denote the vector space of all self-adjoint linear transformations on $\Lambda^{p}$, with inner product $\langle T, U\rangle=\operatorname{tr} T \circ U$. Elements of $\mathscr{R}$ will be called $p$-th curvature operators on $V$. We associate to each $R \in \mathscr{R}$ its $p$-th sectional curvature function $\sigma_{R}: \mathscr{G} \rightarrow R$ defined by $\sigma_{R}(\mathscr{P})=\langle R(\mathscr{P}), \mathscr{P}\rangle$ for $\mathscr{P} \in \mathscr{G}$.

Note. The Plücker coordinates of $\alpha \in \Lambda^{p}$ will be denoted as above by subscripted $\alpha$ 's. A caret $\wedge$ above a symbol indicates that that term or index is to be omitted.

Let a (not necessarily orthonormal) basis $\left\{e_{1}, \cdots, e_{n}\right\}$ be chosen for $V$. The following result is well known. For a proof, see Hodge and Pedoe [1, pp. $309 \mathrm{ff}]$.

Lemma 1.1. (The Grassmann quadratic p-relations). The p-vector $\alpha$ is decomposable if and only if its Plücker coordinates satisfy

$$
\sum_{k=1}^{p+1}(-1)^{k} \alpha_{i_{1} \cdots \hat{i}_{k} \cdots i_{p+1}} \alpha_{i_{k} j_{1} \cdots j_{p-1}}=0
$$

for all $1 \leq i_{k}, j_{m} \leq n$.

For ordered sets $I=\left(i_{1}, \cdots, i_{p+1}\right)$ and $J=\left(j_{1}, \cdots, j_{p-1}\right), 1 \leq i_{k}, j_{m} \leq n$, we define the quadratic form $S_{I J}^{*}$ on $\Lambda^{p}$ by

$$
S_{I J}^{*}(\alpha)=\sum_{k=1}^{p+1}(-1)^{p+k+1} \alpha_{i_{1} \cdots \hat{i}_{k} \cdots i_{p+1}} \alpha_{i_{k} j_{1} \cdots j_{p-1}} .
$$

Clearly $\alpha \in \Lambda^{p}$ is decomposable if and only if $S_{I J}^{*}(\alpha)=0$ for all such $I$ and $J$. Each $S_{I J}^{*}$ will be called a (Grassmann quadratic) $p$-relation. To avoid ambiguity, it may be necessary to write $S_{i_{1} \cdots i_{p+1} j_{1} \cdots j_{p-1}}^{*}$ for $S_{I J}^{*}$.

Let $\mathscr{S}^{*}$ denote the subspace generated by the $p$-relations in the vector space of quadratic forms on $\Lambda^{p}$. Since any $S^{*} \in \mathscr{S}^{*}$ is a linear combination of those given by (2), $S^{*}(\alpha)=0$ for all decomposable $\alpha$. Lemma 1.5 will show that this property characterizes $\mathscr{S}^{*}$, that is, $S^{*}$ is in $\mathscr{S}^{*}$ if and only if $S^{*}(\alpha)=0$ for all decomposable $\alpha$. Lemma 1.4 and the remark preceding it will show that the set $\mathscr{S}^{*}$ is independent of the choice of basis.

The set of $p$-relations $\left\{S_{I J}^{*}\right\}$, which by definition spans $\mathscr{S}^{*}$, is far from linearly independent. In fact, routine computation shows that $S_{I J}^{*}$ is alternating in the sets $I$ and $J$. That is, if $\sigma$ and $\tau$ are permutations of $p+1$ and $p-1$ elements respectively, then

$$
S_{\sigma(I) \tau(J)}^{*}=\varepsilon(\sigma) \varepsilon(\tau) S_{I J}^{*}
$$


where $\varepsilon$ denotes the sign of the permutation. The sets $I$ and $J$ need not be disjoint. It will be shown in $\S 2$ that they may have as many as $p-2$ elements in common without $S_{I J}^{*}$ vanishing. If, however, $J \subset I$, then $S_{I J}^{*}$ is identically zero on $\Lambda^{p}$. To span $\mathscr{S}^{*}$, it therefore suffices to consider only pairs of indexing sets $I$ and $J$ such that $i_{1}<\cdots<i_{p+1}, j_{1}<\cdots<j_{p-1}$, and $\left\{j_{1}, \cdots, j_{p+1}\right\}$ $\not \subset\left\{i_{1}, \cdots, i_{p_{+}}\right\}$. Such a pair of $(p+1)$ - and $(p-1)$-tuples will be said to be standard. A slightly smaller class of sets $I$ and $J$ will index a basis for $\mathscr{S}^{*}$ (§ 2).

Recall that the space of quadratic forms on $\Lambda^{p}$ is naturally isomorphic to the space of homomorphisms $R: \Lambda^{p} \rightarrow\left(\Lambda^{p}\right)^{*}$ such that $(R \xi) \eta=(R \eta) \xi$. An isomorphism $\Phi$ is given by

$$
\left[\left(\Phi R^{*}\right) \xi\right] \eta=R^{*}(\xi+\eta)-R^{*} \xi-R^{*} \eta
$$

for $R^{*}$ a quadratic form on $\Lambda^{p}$ and $\xi, \eta \in \Lambda^{p}$. Let $\mathscr{S}$ be the image of $\mathscr{S}^{*}$ under $\Phi$. We shall denote $\Phi\left(S^{*}\right)$ by $S$. Elements of $\mathscr{S}$ will also be called $p$ relations. To avoid confusion, we may write $\mathscr{S}\left(\Lambda^{p}(V)\right)$ for the $p$-relations on the vector space $V$.

Since $(S \alpha) \alpha=2 S^{*}(\alpha)$, we have

Corollary 1.2. $\alpha \in \Lambda^{p}$ is decomposable if and only if $(S \alpha) \alpha=0$ for all $S \in \mathscr{S}$.

Let $\left\{e_{1}, \cdots, e_{n}\right\}$ be a basis for $V$, and $\left\{e^{1}, \cdots, e^{n}\right\}$ the corresponding dual basis for $V^{*}$. Since $\Lambda^{p}(V)^{*} \cong \Lambda^{p}\left(V^{*}\right)$,

$$
\left\{E^{k}=e^{k_{1}} \wedge \cdots \wedge e^{k_{p}} \mid 1 \leq k_{1}<\cdots<k_{p} \leq n\right\}
$$

is a basis for $\Lambda^{p^{*}}$. Computation shows that the action of $S_{I J}$ on basis $p$-vectors $\left\{E_{k}=e_{k_{1}} \wedge \cdots \wedge e_{k_{p}} \mid 1 \leq k_{1}<\cdots<k_{p} \leq n\right\}$ is

$$
\begin{aligned}
& S_{I J}\left(e_{i_{1}} \wedge \cdots \wedge \hat{e}_{i_{\lambda}} \wedge \cdots \wedge e_{i_{p+1}}\right)=(-1)^{p+\lambda+1} e^{i_{\lambda}} \wedge e^{j_{1}} \wedge \cdots \wedge e^{j_{p-1}}, \\
& S_{I J}\left(e_{i_{\lambda}} \wedge e_{j_{1}} \wedge \cdots \wedge e_{j_{p-1}}\right)=(-1)^{p+\lambda-1} e^{i_{1}} \wedge \cdots \wedge \hat{e}^{i_{\lambda}} \wedge \cdots \wedge e^{i_{p+1}},
\end{aligned}
$$

$S_{I J}$ (other basis vectors) $=0$.

We remark that $S_{I J}$ is zero on any basis $p$-vector whose wedge factors are not chosen from the $e_{i}$ 's and $e_{j}$ 's. Furthermore, if $k \in I \cap J$, then $S_{I J}$ is zero on any basis $p$-vector not containing $e_{k}$ as a wedge factor, and $S_{I J}\left(e_{k} \wedge \eta\right)=$ $e^{k} \wedge \eta^{*}$ for some $\eta^{*} \in \Lambda^{p-1^{*}}$. Therefore, if $S_{I J}$ has $h$ repeating indices $i_{1}, \cdots, i_{h}$, then $\left[S_{I J}\left(e_{\alpha_{1}} \wedge \cdots \wedge e_{\alpha_{p}}\right)\right] e_{\beta_{1}} \wedge \cdots \wedge e_{\beta_{p}}$ is nonzero only if $\left\{\alpha_{1}, \cdots, \alpha_{p}\right\} \cap$ $\left\{\beta_{1}, \cdots, \beta_{p}\right\}=\left\{i_{1}, \cdots, i_{h}\right\}$. Such an $S_{I J}$ is linearly independent of the span of the $p$-relations whose repetitions number other than $h$. Let $\mathscr{S}_{h}$ denote the span of the $p$-relations with $h$ repeating indices.

Let $\left\{E_{i}\right\}$ be an ordered bases for $\Lambda^{p}$ of decomposables of the form $e_{i_{1}} \wedge \cdots \wedge e_{i_{p}}$ with $i_{1}<\ldots<i_{p}$. For $S \in \mathscr{S}$, we consider the symmetric matrix 


$$
\left[s_{i j}=\left(S E_{i}\right) E_{j}\right], \quad 1 \leq i, j \leq \operatorname{dim} \Lambda^{p} .
$$

It is easily shown that nonzero entries for $S \in \mathscr{S}_{h}$ and $S^{\prime} \in \mathscr{S}_{h^{\prime}}\left(h^{\prime} \neq h\right)$ can not appear in the same position in the matrix. We therefore have

Lemma 1.3. $\mathscr{S}$ is the direct sum of the subspaces $\mathscr{S}_{h}$.

When an inner product is defined for $\mathscr{S}$, it will be evident that the subspaces are orthogonal. It would be interesting to know if this decomposition is independent of the choice of basis, but we shall not need this information.

Using the inner product on $\Lambda^{p}(V)$, we now identify the space of homomorphisms $R$ from $\Lambda^{p}$ to $\Lambda^{p^{*}}$ for which $(R \xi) \eta=(R \eta) \xi$ with the space $\mathscr{R}$ of self-adjoint linear transformations on $\Lambda^{p}$ by $\langle R(\xi), \eta\rangle=(R \xi) \eta$. The space of $p$-relations will now be considered a subspace of $\mathscr{R}$. Equations (4) hold with the indices of the right hand side lowered.

Let $\mathscr{B}$, the space of Bianchi transformations, be the orthogonal complement in $\mathscr{R}$ of $\mathscr{S}$. For any $S \in \mathscr{S}$, the condition $\langle R, S\rangle=0$ will be called a Bianchi identity for $R$. The Bianchi identities have appeared previously in [4] but were remarked to be equivalent to the condition Alt $R=0 . R \in \mathscr{B}$ implies Alt $R=0$ but the converse is false [3].

For $p=2, \mathscr{S}$ is spanned by $\left\{S_{i j k l} \mid 1 \leq i<j<k<l \leq n\right\}$, and " $R \in \mathscr{B}$ " is the usual first Bianchi identity for $R$ by Lemma 1.4. The proof of this lemma uses only the fact that $\mathscr{S}$ is the span of the $S_{I J}$ defined by (2) and (3). The lemma gives a condition that $R \in \mathscr{R}$ be orthogonal to $\mathscr{S}$ without reference to a basis, and so we indeed have that $\mathscr{S}$ is independent of the choice of basis.

Lemma 1.4. $R \in \mathscr{R}$ satisfies the Bianchi identities $(R \in \mathscr{B})$ if and only if

$$
\sum_{\lambda=1}^{p+1}(-1)^{\lambda}\left\langle R\left(w_{1} \wedge \cdots \wedge \hat{w}_{\lambda} \wedge \cdots \wedge w_{p+1}\right), w_{\lambda} \wedge w_{p+2} \wedge \cdots \wedge w_{2 p}\right\rangle=0
$$

for all $w_{1}, \cdots, w_{2 p} \in V$.

Note. (5) is often used to define the Bianchi identities, for instance in [2] and [4]. We shall prove this lemma to emphasize the role of the p-relations in this definition.

Proof. Let an orthonormal basis $e_{1}, \cdots, e_{n}$ be chosen for $V$, and let $I, J$ be a standard pair. Then, using (4),

$$
\begin{aligned}
\left\langle R, S_{I J}\right\rangle= & \operatorname{tr} R \circ S_{I J}=\sum_{k}\left\langle R \circ S_{I J}\left(E_{k}\right), E_{k}\right\rangle=\sum_{k}\left\langle R\left(E_{k}\right), S_{I J}\left(E_{k}\right)\right\rangle \\
= & \sum_{\lambda=1}^{p+1}\left[\left\langle R\left(e_{i_{1}} \wedge \cdots \wedge \hat{e}_{i_{\lambda}} \wedge \cdots \wedge e_{i_{p+1}}\right), S_{I J}\left(e_{i_{1}} \wedge \cdots \wedge \hat{e}_{i_{\lambda}} \wedge \cdots \wedge e_{i_{p+1}}\right)\right\rangle\right. \\
& \left.+\left\langle R\left(e_{j_{1}} \wedge \cdots \wedge e_{i_{\lambda}} \wedge \cdots \wedge e_{j_{p-1}}\right), S_{I J}\left(e_{j_{1}} \wedge \cdots \wedge e_{i_{\lambda}} \wedge \cdots \wedge e_{j_{p-1}}\right)\right\rangle\right] \\
= & \sum_{\lambda=1}^{p+1}\left[\left\langle R\left(e_{i_{1}} \wedge \cdots \wedge \hat{e}_{i_{\lambda}} \wedge \cdots \wedge e_{i_{p+1}}\right),(-1)^{p+\lambda+1} e_{i_{\lambda}} \wedge e_{j_{1}} \wedge \cdots \wedge e_{j_{p-1}}\right\rangle\right. \\
& \left.+\left\langle R\left(e_{i_{\lambda}} \wedge e_{j_{1}} \wedge \cdots \wedge e_{j_{p-1}}\right),(-1)^{p+\lambda+1} e_{i_{1}} \wedge \cdots \wedge \hat{e}_{i_{\lambda}} \wedge \cdots \wedge e_{i_{p+1}}\right\rangle\right]
\end{aligned}
$$




$$
\begin{gathered}
=2(-1)^{p+1} \sum_{\lambda=1}^{p+1}(-1)^{\lambda}\langle \\
\quad R\left(e_{i_{1}} \wedge \cdots \wedge \hat{e}_{i_{\lambda}} \wedge \cdots \wedge e_{i_{p+1}}\right) \\
e_{i_{\lambda}} \wedge e_{j_{1}} \wedge \cdots \wedge e_{j_{p-1}}>.
\end{gathered}
$$

Now $R$ is in $\mathscr{B}$ if and only if $\left\langle R, S_{I J}\right\rangle=0$ for all $p$-relations indexed by standard pairs $I, J$, if and only if for all sets $\left\{e_{i_{1}}, \cdots, e_{i_{p+1}}, e_{j_{1}}, \cdots, e_{j_{p-1}}\right\}$ of basis vectors for $V$,

$$
\sum_{\lambda=1}^{p+1}(-1)^{\lambda}\left\langle R\left(e_{i_{\mathbf{1}}} \wedge \cdots \wedge \hat{e}_{i_{\lambda}} \wedge \cdots \wedge e_{i_{p+1}}\right), e_{i_{\lambda}} \wedge e_{j_{1}} \wedge \cdots \wedge e_{j_{p-1}}\right\rangle=0 .
$$

This is equivalent to (5) by the linearity of $R$ and $\langle$,$\rangle . q.e.d.$

For $R \in \mathscr{R}$ we consider its sectional curvature $\sigma_{R}$.

Lemma 1.5. If $R \in \mathscr{B}$ and $\sigma_{R} \equiv 0$, then $R=0$.

Remark. This lemma is a generalization of the well known fact that in the presence of the Bianchi identity, sectional curvature determines the curvature operator. For a proof in this case, see [2] or [3].

The following corollary shows that vanishing sectional curvature characterizes $\mathscr{S}$.

Corollary 1.6. A curvature operator $R$ is a p-relation $(R \in \mathscr{S})$ if and only if $\sigma_{R} \equiv 0$.

Proof. It is clear from Corollary 1.2 that the sectional curvature of every operator in $\mathscr{S}$ is identically zero. Conversely, let $R=R_{1}+S$ where $R_{1} \in \mathscr{B}$ and $S \in \mathscr{S}$. Then $\sigma_{R_{1}}=\sigma_{R}-\sigma_{S} \equiv 0$ and so $R_{1}=0$ by Lemma 1.5 , and therefore $R \in \mathscr{S}$.

Remark. Notice that the identity map $I$, which has sectional curvature 1, lies in the subspace $\mathscr{B}$, for if $\left\{E_{i}\right\}$ is an orthonormal basis for $\Lambda^{p}$ of decomposable $p$-vectors,

$$
\langle S, I\rangle=\sum_{i}\left\langle S \circ I\left(E_{i}\right), E_{i}\right\rangle=\sum_{i} \sigma_{S}\left(E_{i}\right)=0
$$

for all $S \in \mathscr{S}$. Therefore if $R$ satisfies Bianchi, so does $R+\lambda I$ for all real numbers $\lambda$.

\section{A basis for the $p$-relations}

For the computations in $\S 3$ we shall need to know a basis for $\mathscr{S}$, and so we give a proof of

Theorem 2.1. The set of p-relations

$$
\left\{S_{I J} \mid I, J \text { is a standard pair and } i_{p+1} \leq j_{p-1}\right\}
$$

is a basis for $\mathscr{S}$.

For this discussion, we view $\mathscr{S}$ as a subset of $\operatorname{Hom}\left(\Lambda^{p}, \Lambda^{p^{*}}\right)$. By a series of lemmas, we shall find a basis for $\mathscr{S}_{0}$ in the case $n=2 p$, and use this to 
find a basis for $\mathscr{S}_{0}$ in general. We shall then show how each $p$-relation with $h$ repeated indices corresponds to a $(p-h)$-relation with distinct indices and the theorem will follow. The decomposition of $\mathscr{S}$ as the direct sum of the $\mathscr{S}_{h}$ is crucial.

If $J=\left(j_{1}, \cdots, j_{p-1}\right)$ with $j_{1}<\cdots<j_{p-1} \leq 2 p$, let $\varepsilon(J)$ denote the sign of the permutation

$$
(1, \cdots, 2 p) \rightarrow\left(1, \cdots \hat{j}_{1}, \cdots, \hat{j}_{p-1}, \cdots, 2 p, j_{1}, \cdots, j_{p-1}\right) .
$$

Let $S^{J}$ denote the $p$-relation $\varepsilon(J) S_{1 \cdots \hat{j}_{1} \cdots \hat{j}_{p-1} \cdots(2 p) j_{1} \cdots j_{p-1}}$.

Lemma 2.2. If $n=2 p$, then $\mathscr{S}_{0}$ is spanned by

$$
\Pi=\left\{S^{J} \mid j_{1}<\cdots<j_{p-1}=2 p\right\} .
$$

Remark. We include the factor $\varepsilon(J)$ so that if $S \in \Pi$ and $E$ is a basis vector for $\Lambda^{p}$, then $S(E) * E=0$ or +1 , where $*$ denotes the Hodge star operator.

Proof of Lemma 2.2. It has been shown that $\mathscr{S}_{0}$ is spanned by the set of $S_{I J}$ such that $I, J$ is a standard pair and $I \cup J=\{1, \cdots, 2 p\}$, since the $2 p$ indices of $S$ are distinct and all $\leq n=2 p$. Each such $S$ is determined by the set $J$ of its last $p-1$ indices, and so $\mathscr{S}_{0}$ is spanned by the $C_{2 p, p-1}$ relations in the set

$$
\tilde{\Pi}=\left\{S^{J} \mid j_{1}<\cdots<j_{p-1} \leq 2 p\right\} .
$$

We shall show that we can span $\mathscr{S}_{0}$ by requiring that $j_{p-1}=2 p$.

As above, let $\Pi \subset \tilde{\Pi}$ be the $p$-relation $S^{J}$ for which $j_{p-1}=2 p$. Any $S$ which is in $\tilde{I}$ but not in $\Pi$ is of the form $\pm S_{\nu_{1} \cdots \nu_{p}(2 p) \mu_{1} \cdots \mu_{p-1}}$. For such an $S$ and for $\lambda=0,1, \cdots, p-2$, let

$$
\Pi^{\lambda}(S)=\left\{S^{J} \in \Pi \mid \#\left(J \cap\left\{\mu_{1}, \cdots \mu_{p-1}\right\}\right)=\lambda\right\} .
$$

Then we claim that

$$
S=\sum_{\lambda=0}^{p-2} \sum_{T \in \Pi^{\lambda}(S)} c_{\lambda} T
$$

where $c_{0}=\frac{1}{p-1}$ and $c_{\lambda}=-\left(\frac{\lambda}{p-\lambda-1}\right) c_{\lambda-1}$ for $\lambda \geq 1$. This will establish the fact that $\Pi$ spans $\mathscr{S}_{0}$.

Let $*: \Lambda^{p} \rightarrow \Lambda^{p}$ denote the Hodge star operator defined by

$$
\left\langle^{*} \alpha, \beta\right\rangle e_{1} \wedge e_{2} \wedge \cdots \wedge e_{2 p}=\alpha \wedge \beta
$$

for $\alpha, \beta \in \Lambda^{p}$. Up to sign, $*$ takes basis elements to basis elements.

We write the basis elements for $A^{p}$ in the following order: first we list the basis elements $e_{k_{1}} \wedge \cdots \wedge e_{k_{p-1}} \wedge e_{2 p}$ containing $e_{2 p}$ as a wedge factor, in 
numerical order of $k_{1} \cdots k_{p-1}$. Next we list $*\left(e_{k_{1}} \wedge \cdots \wedge e_{k_{p-1}} \wedge e_{2 p}\right)$, also in numerical order of $k_{1} \cdots k_{p-1}$. Let $E_{i}$ stand for the $i$-th basis vector in this ordering. We have listed all of the basis vectors for $\Lambda^{p}$ since $E_{i}$ contains $e_{2 p}$ as a wedge factor if and only if $* E_{i}$ does not.

With this ordering of the basis vectors, we consider the matrix $S\left(E_{i}\right) E_{j}$. Letting $\delta_{i}(S)=S\left(E_{i}\right)^{*} E_{i}$, any $S \in \tilde{\Pi}$ and therefore any $S \in \mathscr{S}_{0}$ has matrix.

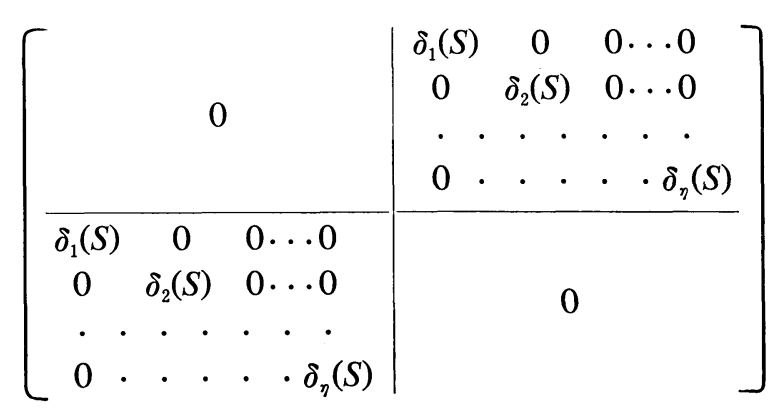

and $S$ is determined by the $\eta=\frac{1}{2} C_{2 p}$, -tuple $\left(\delta_{1}(S), \cdots, \delta_{\eta}(S)\right)$. Notice that the signs for the $p$-relations in $\Pi$ were chosen so that $\delta_{i}(S)=0$ or +1 for $S \in \Pi$.

We shall prove (6) by showing that for each $i$, the coordinates $\delta_{i}$ of both sides are equal.

If $i \leq \eta$, let $K^{i}$ denote the set $\left\{k_{1}, \cdots, k_{p-1}\right\}$ for which $E_{i}=e_{k_{1}} \wedge \cdots \wedge$ $e_{k_{p-1}} \wedge e_{2 p}$. The only $T \in \Pi$ for which $\delta_{i}(T) \neq 0$ are the $p-1$ relations

$$
\left\{S^{\beta}=S^{k_{1} \cdots \hat{i}_{\beta} \cdots k_{p-12 p}} \in \Pi\right\} \text {. }
$$

We shall find the coordinate $\delta_{i}\left(S_{\nu_{1} \cdots \nu_{p}(2 p) \mu_{1} \cdots \mu_{p-1}}\right)$, depending on the number of elements in $\left\{\mu_{1}, \cdots, \mu_{p-1}\right\} \cap K^{i}$.

Case 0: $\left\{\mu_{1}, \cdots, \mu_{p-1}\right\} \cap K^{i}=\emptyset$. Let $\gamma$ denote both the ordered set $\left(\nu, k_{1}, \cdots, k_{p-1}, 2 p, \mu_{1}, \cdots, \mu_{p-1}\right)$ and the permutation of $(1,2, \cdots, 2 p)$ given by the ordered set. Any $S \in \tilde{\Pi}-\Pi$ which falls into case 0 is of the form $\varepsilon(\gamma) S_{\gamma}$ for some $\gamma$ (i.e., for some $\nu$ ), and

$$
\begin{aligned}
\delta_{i}(S) & =\varepsilon(\gamma)\left[S_{\gamma}\left(E_{i}\right)\right] * E_{i}=\varepsilon(\gamma)\left[S_{\nu k_{1} \cdots k_{p-1}(2 p) \mu_{1} \cdots \mu_{p-1}}\left(E_{i}\right)\right] * E_{i} \\
& =(-1)^{p} \varepsilon(\gamma)\left[S_{k_{1} \cdots k_{p-1}(2 p) \nu \mu_{1} \cdots \mu_{p-1}}\left(E_{i}\right)\right] * E_{i}=(-1)^{2 p}[\varepsilon(\gamma)]^{2}=1
\end{aligned}
$$

by (4) since $\left[S\left(E_{i}\right)\right] * E_{i}=\left[S\left(* E_{i}\right)\right] E_{i}$.

Now $\delta_{i}(T)=0$ for all $T \in \Pi^{\lambda}(S)$ whenever $\lambda>0$ (in case 0 , the last $p-1$ indices of $S$ have no indices in common with the wedge factor of $E_{i}$ ), and so $\delta_{i}$ on the left hand side of (6) reduces to

$$
\sum_{T \in \Pi^{0}(S)} c_{0} \delta_{i}(T)=\sum_{T \in \Pi^{0}(S)} c_{0}=1=\delta_{i}(S) .
$$

Case $m(0<m<p-1):\left\{\mu_{1}, \cdots, \mu_{p-1}\right\} \cap K^{i}=\left\{k_{\varphi_{1}}, \cdots, k_{\varphi_{m}}\right\}$. We again group common indices (here $k_{\varphi_{1}}, \cdots, k_{\varphi_{m}}$ ) together at the beginning of the last 
$p-1$ slots of $S$. Let $\gamma=\left(\nu_{1}, \cdots, \nu_{m+1}, k_{1}, \cdots, \hat{k}_{\varphi_{1}}, \ldots, \hat{k}_{\varphi_{m}}, \cdots, k_{p-1}, 2 p, k_{\varphi_{1}}\right.$, $\left.\cdots, k_{\varphi_{m}}, \mu_{1}, \cdots, \hat{k}_{\varphi_{1}}, \cdots, \hat{k}_{\varphi_{m}}, \cdots, \mu_{p-1}\right)$, and let $S=\varepsilon(\gamma) S_{r}$. By $(4), S\left(E_{i}\right)=$ $S\left(e_{k_{1}} \wedge \cdots \wedge e_{k_{p-1}} \wedge e_{2 p}\right)=0$ and so $\delta_{i}(S)=0$.

Of the $p-1$ relations $S^{\beta}$ for which $\delta_{i}\left(S^{\beta}\right)=1$, the $m$ relations $S^{\varphi_{h}}$ are in $\Pi^{m-1}(S)$ and the other $p-m-1$ relations $S^{\beta}\left(\beta \neq \varphi_{h}\right)$ are in $\Pi^{m}(S)$. Thus $\delta_{i}$ of the left hand side of (6) is

$$
\begin{aligned}
\sum_{\lambda=m-1}^{m} \sum_{T \in \Pi^{\lambda}(S)} c_{\lambda} \delta_{i}(T) & =c_{m-1} \sum_{h=1}^{m} \delta_{i}\left(S^{\varphi_{h}}\right)+c_{m} \sum_{\beta \neq \varphi_{h}} \delta_{i}\left(S^{\beta}\right) \\
& =m c_{m-1}+(p-m-1) c_{m}=0=\delta_{i}(S) .
\end{aligned}
$$

Case $p-1:\left\{\mu_{1}, \cdots, \mu_{p-1}\right\}=\left\{k_{1}, \cdots, k_{p-1}\right\}$. Letting $\gamma=\left\{\nu_{1}, \cdots \nu_{p}, 2 p\right.$, $\left.k_{1}, \cdots, k_{p-1}\right)$, we have $S=\varepsilon(\gamma) S_{r}$ and

$$
\begin{aligned}
\delta_{i}(S) & =\left[S\left(E_{i}\right)\right] * E_{i}=\varepsilon(\gamma)\left[S_{\nu_{1} \cdots \nu_{p}(2 p) k_{1} \cdots k_{p-1}}\left(E_{i}\right)\right] * E_{i} \\
& =(-1)^{p}[\varepsilon(\gamma)]^{2}=(-1)^{p}
\end{aligned}
$$

by (4).

All $S^{\beta}$ such that $\delta_{i}\left(S^{\beta}\right)=1$ are in $\Pi^{p-2}(S)$, and $\delta_{i}$ of the left hand side of (6) is

$$
\sum_{T \in \Pi^{p-2(S)}} c_{p-2} \delta_{i}(T)=(p-1) c_{p-2}=(-1)^{p-2}=\delta_{i}(S) .
$$

This finishes the proof of Lemma 2.2. q.e.d.

If the members of $\Pi$ are linearly dependent, there must exist real numbers $a_{j_{1} \ldots j_{p-2}}$, not all zero, so that

$$
\sum_{j_{1}<\cdots<j_{p-2}} a_{j_{1} \cdots j_{p-2}} S^{j_{1} \cdots j_{p-2}}=0 .
$$

If $E_{i}$ or $* E_{i}$ is $e_{k_{1}} \wedge \cdots \wedge e_{k_{p-1}} \wedge e_{2 p}$, taking $\delta_{i}$ of both sides of (7) gives

$$
\sum_{\lambda=1}^{p-1} a_{k_{1} \cdots \hat{\lambda}_{\lambda} \cdots k_{p-1}}=0 .
$$

Since $S \in \mathscr{S}_{0}$ is determined by $\left\{\delta_{i}(S)\right\}$, (7) is equivalent to (8) for all sets $k_{1}, \cdots, k_{p-1}$ such that $1 \leq k_{1}<\cdots<k_{p-1}<2 p$. The next lemma, due to C. H. Sah, shows that the system (8) of homogeneous equations has no nontrivial solutions.

Lemma 2.3. Let $I$ be a finite set with $q$ elements, and let $r \geq 1$ be an integer such that $q \geq 2 r-1$. For each $(r-1)$-element subset $J$ of $I$, let $a_{J}$ be in a field of characteristic zero. If, for each $r$-element subset $K$ of $I$, $\sum_{J \subset K} a_{J}=0$, then all $a_{J}=0$.

Proof. By induction. When $r=1$, there is only one $a_{\emptyset}$ and at least one equation $a_{\emptyset}=0$. 
We assume that the lemma is valid for $r$. Let $I^{\prime \prime}$ be a finite subset with $q \geq 2 r+1$ elements. Let $I^{\prime}$ be any subset of $I^{\prime \prime}$ with $2 r+1$ elements. Set $I^{\prime}=I \cup\{ \pm r\}$ where $I=\{0, \pm 1, \cdots, \pm(r-1)\}$. The subsystems (for $K \subseteq I$, $\sharp(K)=r)$

$$
\sum_{J \subset K} a_{J \cup\{r\}}=-a_{K} \text { and } \sum_{J \subset K} a_{J \cup\{-r\}}=-a_{K}
$$

have at most one solution (by the induction hypothesis) depending only on the $a_{K}$ 's. Thus $a_{J \cup\{r\}}=a_{J \cup\{-r]}$, i.e., for any two subsets $L$ and $L^{\prime}$ of $I^{\prime \prime}$ with \#(L) $=\#\left(L^{\prime}\right)=r$ and $\#\left(L \cap L^{\prime}\right)=r-1$, we have $a_{L}=a_{L^{\prime}}$. Since any two $r$-element subsets of $I^{\prime \prime}$ can be connected by a sequence of subsets such that consecutive pairs have $r-1$ elements in common, $a_{L}=$ constant. Since the coefficients of the system are +1 (i.e., nonnegative), any one of the equations now implies that all $a_{L}$ are zero.

Corollary 2.4. If $n=2 p$, the set $\Pi$ of p-relations is a basis for $\mathscr{S}_{0}$.

Proof. If $K \subset I=\{1, \cdots, 2 p\}$ has $r=p-1$ elements, (8) becomes

$$
\sum_{\substack{J \subset K \\ \#(J)=r-1}} a_{J}=0
$$

Taking $q=2 p$, Lemma 2.3 implies that $a_{J}=0$ for all $J \subset I$ such that $\#(J)=$ $p-2$. Therefore (7) implies $a_{j_{1} \cdots j_{p-2}}=0$ for all $j_{1}<\cdots<j_{p-2}$, i.e., $\left\{S^{j_{1} \cdots j_{p-2}}\right\}$ is independent. q.e.d.

The independence of this set of $p$-relations implies the independence of each of the spanning sets which will be found in Lemmas 2.5, 2.6 and 2.7, and therefore in Theorem 2.1.

If $U \subset V, \mathscr{L} U$ will denote the linear span of the set $U$.

Lemma 2.5. The set

$$
\Pi_{0}=\left\{S_{I J} \mid \boldsymbol{I}, \boldsymbol{J} \text { is a standard pair such that } I \cap J=\emptyset \text { and } i_{p+1}<j_{p-1}\right\}
$$

is a basis for $\mathscr{S}_{0}$.

Proof. If $n<2 p$, then $\mathscr{S}_{0}=\{0\}$ and so we are left with the case $n>2 p$.

For each subspace $W$ spanned by $2 p$ basis vectors $\left\{e_{i_{1}}, \cdots, e_{i_{2 p}} \mid i_{1}<\cdots<i_{2 p}\right\}$, the relations

$$
\left\{S^{i_{j_{1}} \cdots i_{j_{p-2}}}=S_{i_{1} \cdots \hat{i}_{j_{1}} \cdots \hat{i}_{j_{p-2}} \cdots i_{2 p-1} i_{j_{1}} \cdots i_{j_{p-2}} i_{2 p}}\right\}
$$

form a basis for $\mathscr{S}_{0}\left(\Lambda^{p}(W)\right)$ by Lemma 2.2.

For $S \in \mathscr{S}_{0}\left(\Lambda^{p}(W)\right),\left[S\left(e_{j_{1}} \wedge \cdots \wedge e_{j_{p}}\right)\right] e_{k_{1}} \wedge \cdots \wedge e_{k_{p}}$ is nonzero only if $\left\{j_{1}, \cdots, j_{p}, k_{1}, \cdots, k_{p}\right\}=\left\{i_{1}, \cdots, i_{2 p}\right\}$, and so each of the basis relations for $\mathscr{S}^{0}\left(\Lambda^{p}(W)\right)$ is independent of the collection of spanning relations for all $\mathscr{S}_{0}\left(\Lambda^{p}\left(W^{\prime}\right)\right)$ where $W^{\prime}=\mathscr{L}\left\{e_{\lambda_{1}}, \cdots, e_{\lambda_{2} p}\right\} \neq W$. Since every $S_{I J} \in \mathscr{S}_{0}\left(\Lambda^{p}(V)\right)$ is in $\mathscr{S}_{0}\left(\Lambda^{p}(W)\right)$ for $W=\mathscr{L}\left\{e_{i_{1}}, \cdots, e_{i_{p+1}}, e_{j_{1}}, \cdots, e_{j_{p-1}}\right\}$, we conclude that $\mathscr{S}_{0}$ is the direct sum $\Sigma \oplus \mathscr{S}_{0}\left(\Lambda^{p}(W)\right)$ as $W$ ranges over subspaces spanned by 
$2 p$ basis vectors for $V$, and that $\Pi_{0}$ is a basis for $\mathscr{S}_{0}$. The dimension of $\mathscr{S}_{0}$ is $C_{n, 2 p} C_{2 p-1, p-2}$.

Lemma 2.5. The set

$$
\begin{aligned}
\Pi_{1}=\left\{S_{k i_{1} \cdots i_{p} j_{1} \cdots j_{p-2} k} \mid i_{1}<\cdots<i_{p}<j_{p-2}, j_{1}<\cdots<j_{p-2},\right. \\
\left.\quad k \notin\left\{i_{1}, \cdots, i_{p}, j_{1}, \cdots, j_{p-2}\right\},\left\{i_{1}, \cdots, i_{p}\right\} \cap\left\{j_{1}, \cdots, j_{p-2}\right\}=\emptyset\right\}
\end{aligned}
$$

is a basis for $\mathscr{S}_{1}$, the span of the p-relations with one repeated index.

Proof. $\quad \mathscr{S}_{1}$ is the direct sum of the subspaces $\mathscr{S}_{1}^{k}$, the span of the $p$-relations whose repeated index is $k$. For if $S \in \mathscr{S}_{1}^{k},\left[S\left(e_{j_{1}} \wedge \cdots \wedge e_{j_{p}}\right)\right] e_{k_{1}} \wedge \cdots \wedge e_{k_{p}}$ is nonzero only if $\left\{j_{1}, \cdots, j_{p}\right\} \cap\left\{k_{1}, \cdots, k_{p}\right\}=\{k\}$ by (4). Similarly, $\mathscr{S}_{1}^{k}$ is further the direct sum of $\mathscr{S}_{1}^{k ; I}$, the span of the $p$-relations whose non-repeating indices are $I=\left\{i_{1}, \cdots, i_{2 p_{-2}}\right\}$.

We now find a basis for $\mathscr{S}_{1}^{k ; I}$. Let $W^{I}$ be the $(2 p-2)$-dimensional subspace of $V$ spanned by $\left\{e_{i} \mid i \in I\right\}$. Let $S=S_{k \nu_{1} \cdots \nu_{p} \mu_{1} \cdots \mu_{p-2} k} \in \mathscr{S}_{1}^{k ; I}$. Then the $\nu^{\prime}$ 's and $\mu$ 's are chosen from $I$. When $E$ is in the chosen basis for $\Lambda^{p}(V), S(E)=0$ if $E$ does not contain $e_{k}$ as a wedge factor, or if $E=e_{k} \wedge E^{\prime}$ but $E^{\prime} \notin \Lambda^{p-1}\left(W^{I}\right)$. Let $\hat{S}=\hat{S}_{\nu_{1} \cdots \nu_{p} \mu_{1} \cdots \mu_{p-2}}$ be the obvious $(p-1)$-relation on $W^{I}$. If $E=e_{k} \wedge E^{\prime}$ where $E^{\prime} \in \Lambda^{p-1}\left(W^{I}\right)$, then $S(E)=\hat{S}\left(E^{\prime}\right) \wedge e_{k}$ by (4). For fixed $k$, the correspondence $S \in \mathscr{S}_{1}^{k ; I}\left(\Lambda^{p}(V)\right) \rightarrow \hat{S} \in \mathscr{S}_{0}\left(\Lambda^{p-1}\left(W^{I}\right)\right)$ is clearly an isomorphism. Therefore the $C_{2 p-3, p-3}$ basis relations for $\mathscr{S}_{0}\left(\Lambda^{p-1}\left(W^{I}\right)\right)$ give rise to an equal number of basis relations for $\mathscr{S}_{1}^{k ; I}$, namely $\left\{S_{k i_{1} \cdots i_{2 p-2} k} \mid i_{1}<\cdots<i_{p}<\right.$ $\left.i_{2 p-2}, i_{p+1}<\cdots<i_{2 p-2}\right\}$. Therefore the $C_{n, 1} C_{n-1,2 p-2} C_{2 p-3, p-3}$ relations in the set $\Pi_{1}$ form a basis for $\mathscr{S}_{1}\left(\Lambda^{p}(V)\right)=\sum_{k, I} \oplus \mathscr{S}_{1}^{k ; I}$.

Lemma 2.7. $A$ basis for $\mathscr{S}_{h}\left(\Lambda^{p}(V)\right)$ is

$$
\begin{aligned}
\Pi_{h}=\left\{S_{k_{1} \cdots k_{h} i_{1} \cdots i_{2 p-2 h} k_{1} \cdots k_{h}} \mid k_{1}<\cdots<k_{h},\right. \\
\\
i_{1}<\cdots<i_{p-h+1}<i_{2 p-2 h}, i_{p-h+2}<\cdots<i_{2 p-2 h}, \\
\left\{i_{1}, \cdots, i_{p-h+1}\right\} \cap\left\{i_{p-h+2}, \cdots, i_{2 p-2 h}\right\}=\emptyset \text { and } \\
\left.\left\{k_{1}, \cdots, k_{h}\right\} \cap\left\{i_{1}, \cdots, i_{2 p-2 h}\right\}=\emptyset\right\} .
\end{aligned}
$$

Proof. An argument identical to that of Lemma 2.6 shows that

$$
\mathscr{S}_{h}\left(\Lambda^{p}(V)\right)=\sum_{K, I} \oplus \mathscr{S}_{h}^{K ; I}
$$

where for fixed $K=\left\{k_{1}, \cdots, k_{h}\right\}$ each element of $\mathscr{S}_{h}^{K}, I\left(I=\left\{i_{1}, \cdots, i_{2 p-2 h}\right\}\right)$ corresponds to an element of $\mathscr{S}_{0}\left(\Lambda^{p-h}\left(W^{I}\right)\right), W^{I}=\mathscr{L}\left\{e_{i} \mid i \in I\right\}$, and the lemma follows. q.e.d.

Since $\mathscr{S}$ is the direct sum of the $\mathscr{S}_{h}(h=0, \cdots, p-2)$ and $\cup \Pi_{h}$ is the set of $p$-relations in Theorem 2.1, the theorem has been proved. The dimension of $\mathscr{S}$ is $\sum_{n=0}^{p-2} C_{n, h} C_{n-h, 2 p-2 h} C_{2 p-2 h-1, p-h-2}$. Translated back to Plücker corrdinates, Theorem 2.1 is Theorem $\mathrm{A}$ of the introduction. 


\section{The main theorem}

In this section we shall show that for any $R \in \mathscr{R}$, the set of points of $\mathscr{G}$ where $\sigma_{R}$ assumes its maximum or minimum is the intersection with $\mathscr{G}$ of a linear subspace of $\Lambda^{p}(V)$. We shall consider the zero set $Z(R)=\left\{\mathscr{P} \in \mathscr{G} \mid \sigma_{R}(\mathscr{P})\right.$ $=0\}$ of an operator $R$ with nonnegative sectional curvature, for the general case is an easy corollary of this one.

As in [5], we first show that if $\mathscr{P}$ is a critical point of $\sigma_{R}$ and $\sigma_{R}(\mathscr{P})=0$, then there exists $S \in \mathscr{S}$, in fact a unique $S$ orthogonal to the $p$-relations which annihilate $\mathscr{P}$, such that $R(\mathscr{P})=S(\mathscr{P})$. Our main contribution is Theorem 3.6 which uses the basis for $\mathscr{S}$ to show that if $\sigma_{R} \geq 0$ and $\sigma_{R}(\mathscr{P})=\sigma_{R}(Q)=0$, then there exists $S \in \mathscr{S}$ such that $\{\mathscr{P}, Q\} \subset \operatorname{Ker}(R-S)$. The finite dimensionality of $\Lambda^{p}$ guarantees the existence of an $S \in \mathscr{S}$ so that all of the zeros of $\sigma_{R}$ are contained in $\operatorname{Ker}(R-S)$. It is immediate that the zeros of $R$ contain $\mathscr{G} \cap \operatorname{Ker}(R-S)$.

Remark. Wherever possible, we refer the reader to [5] for proofs, in particular for the next five. The proofs of Lemma 3.1 and Proposition 3.2 found in [5] for $p=2$ must be generalized to fit our needs. With the help of the results of $\S \S 1$ and 2 , the obvious generalization works.

Lemma 3.1. Let $\mathscr{P} \in \mathscr{G}$. Then $\{\mathscr{P}\} \cup\{S(\mathscr{P}) \mid S \in \mathscr{S}\}$ spans the normal space $N_{\mathscr{P}}$ to $\mathscr{G} \subset \Lambda^{p}$ at $\mathscr{P}$.

We remark that if an orthonormal basis $\left\{e_{1}, \cdots, e_{n}\right\}$ is chosen for $V$ so that $\mathscr{P}=e_{1} \wedge \cdots \wedge e_{p}$, then $N_{\mathscr{g}}$ is spanned by $\mathscr{P}$ and the planes $Q$ of the form $e_{i_{1}} \wedge \cdots \wedge e_{i_{p}}$ such that $\mathscr{P}+Q$ is not decomposable.

Proposition 3.2. Let $R \in \mathscr{R}$, and suppose $\mathscr{P} \in \mathscr{G}$ is a critical zero of $\sigma_{R}$. Then there exists $S \in \mathscr{S}$ such that $\mathscr{P} \in \operatorname{Ker}(R-S)$.

In fact, if a basis for $V$ is chosen as above, there exist unique $\lambda_{I J} \in \boldsymbol{R}$ so that $S=\sum_{I, J} \lambda_{I J} S_{I J}$, where the sum runs only over those $S_{I J}$ in the basis for $\mathscr{S}$ and $I=(1, \cdots, p, i)$.

As in [5] we let $\mathscr{Y}(Z)$ denote those $p$-relations which annihilate $Z \subset \mathscr{G}$, and the superscript $\perp$ denote orthogonal complementation in $\mathscr{S}$.

Lemma 3.3. Let $R \in \mathscr{R}$ and $Z \subset \mathscr{G}$. If there exists $S \in \mathscr{S}$ such that $Z \subset$ $\operatorname{Ker}(R-S)$, then there exists a unique $S_{0} \in \mathfrak{Y}(Z)^{\perp}$ such that $Z \subset \operatorname{Ker}\left(R-S_{0}\right)$. For any $S \in \mathscr{S}, Z \subset \operatorname{Ker}(R-S) \Leftrightarrow S_{0}$ is the orthogonal projection of $S$ onto $\mathfrak{U}(Z)^{\perp}$.

Corollary 3.4. If $\mathscr{P}$ is a critical zero of $\sigma_{R}$, there exists a unique $S_{\mathscr{g}} \in \mathfrak{A}(\mathscr{P})^{\perp}$ such that $\mathscr{P} \in \operatorname{Ker}\left(R-S_{\mathscr{P}}\right)$.

Corollary 3.5. If there exists $S \in \mathscr{S}$ such that $Z(R) \subset \mathscr{G} \cap \operatorname{Ker}(R-S)$, there exists a unique $S_{0} \in \mathfrak{Y}(Z(R))^{\perp}$ such that $Z(R)=\mathscr{G} \cap \operatorname{Ker}\left(R-S_{0}\right)$.

The proof of the main theorem now depends on the existence of an $S \in \mathscr{S}$ which "works" simultaneously for any two planes in $Z(R)$.

Theoerm 3.6. Suppose $R \in \mathscr{R}$ is such that $\sigma_{R} \geq 0$. If $\mathscr{P}, Q \in Z(R)$, then there exists $S \in \mathscr{S}$ such that $\{\mathscr{P}, Q\} \subset \operatorname{Ker}(R-S)$. 
Proof. We assume that $\mathscr{P}$ and $Q$ are linearly independent in $A^{p}(V)$ (otherwise $\mathscr{P}= \pm Q$ and we apply Proposition 3.2). Let $S_{\mathscr{g}}$ (respectively $S_{Q}$ ) denote any curvature operator in $\mathscr{S}$ such that $S_{\mathscr{P}}(\mathscr{P})=R(\mathscr{P})\left[S_{Q}(Q)=R(Q)\right]$, the existence of which is guaranteed by Proposition 3.2. We must find $S \in \mathscr{S}$ whose orthogonal projections onto $\mathfrak{U}(\mathscr{P})^{\perp}$ and $\mathfrak{U}(Q)^{\perp}$ are those of $S_{\mathscr{P}}$ and $S_{Q}$, respectively. Such an $S$ exists if and only if $S_{\mathscr{g}}$ and $S_{Q}$ have the same orthogonal projections onto $[\mathfrak{U}(\mathscr{P})+\mathfrak{U}(Q)]^{\perp}=\mathfrak{U}(\mathscr{P})^{\perp} \cap \mathfrak{U}(Q)^{\perp}$. We shall show that they do through a series of lemmas.

Lemma A. $\mathscr{P}+Q$ is decomposable $\Leftrightarrow \operatorname{dim}(\mathscr{P} \cap Q)=p-1$.

Proof of Lemma A. If $\operatorname{dim}(\mathscr{P} \cap Q)=p-1$, there is a basis $\left\{e_{1}, \cdots, e_{n}\right\}$ for $V$ (not necessarily orthogonal) such that $\mathscr{P}=e_{1} \wedge \cdots \wedge e_{p}$ and $Q=$ $e_{1} \wedge \cdots \wedge e_{p-1} \wedge e_{p+1}$. Then $\mathscr{P}+Q=e_{1} \wedge \cdots \wedge e_{p-1} \wedge\left(e_{p}+e_{p+1}\right)$, which is decomposable.

Assuming that $\mathscr{P} \neq \pm Q$, if $\operatorname{dim}(\mathscr{P} \cap Q) \neq p-1$, let $k=\operatorname{dim}(\mathscr{P} \cap Q)$ $\leq p-2$. There is a basis $\left\{e_{1}, \cdots, e_{n}\right\}$ for $V$ such that $\mathscr{P}=e_{1} \wedge \cdots \wedge e_{p}$ and $Q=e_{1} \wedge \cdots \wedge e_{k} \wedge e_{p+1} \wedge \cdots \wedge e_{2 p-k}$. But $S_{1 \cdots(2 p-k) 1 \cdots k}^{*}(\mathscr{P}+Q)=$ $(-1)^{k(p-k)} \neq 0$ and so $\mathscr{P}+Q$ is not decomposable.

Case 1: $\mathscr{P}+Q$ not decomposable. To prove the theorem in this case, we shall show that $[\mathfrak{U}(\mathscr{P})+\mathfrak{U}(Q)]^{\perp}$ is one-dimensional and find a basis for it.

Lemma B. If $\mathscr{P}+Q$ is not decomposable, then the codimension in $\mathscr{S}$ of $\mathfrak{Y}(\mathscr{P})+\mathfrak{U}(Q)$ is at most 1 .

Proof of Lemma B. We shall want to find the set $\mathfrak{U}(\mathscr{P})$ explicitly and without reference to the inner product on $\Lambda^{p}$. For this lemma only we regard $\mathscr{S}$ as a subset of Hom $\left(\Lambda^{p}, \Lambda^{p^{*}}\right)$ and observe that $S \in \mathfrak{X}(\mathscr{P}) \Leftrightarrow S(\mathscr{P})=0 \in \Lambda^{p^{*}}$.

Consider the basis $p$-relations $S_{I J}$ (Theorem 2.1). Most of the basis $p$-relations are in either $\mathfrak{A}(\mathscr{P})$ or $\mathfrak{A}(Q)$, and for all the others, taken pairwise their linear span intersects $\mathfrak{U}(\mathscr{P}) \cap \mathfrak{U}(Q)$ nontrivially.

An elimination argument involving (4) shows that the only basis $p$-relations which are in neither $\mathfrak{U}(\mathscr{P})$ nor $\mathfrak{U}(Q)$ are $S_{1 \cdots p i 1 \cdots k(p+1) \cdots \hat{i} \cdots(2 p-k)}$ where $i \epsilon$ $\{p+1, \cdots, 2 p-k\}$. For $1 \leq h \leq p-k$, let $S^{h}=S_{1 \cdots p i 1 \cdots k(p+1) \cdots \hat{i} \cdots(2 p-k)}$ where $i=p+h$. We have $S^{h}(\mathscr{P})=(-1)^{k+h-1} Q^{*}$ where $Q^{*} \in \Lambda^{p^{*}}$ is dual to $Q$. Similarly, $S^{h}(Q)=(-1)^{k+h-1} \mathscr{P} *$. Now $\left[S^{1}+(-1)^{h} S^{h}\right] \mathscr{P}=\left[S^{1}+(-1)^{h} S^{h}\right] Q$ $=0$. Therefore for each $h \geq 2, S^{1}+(-1)^{h} S^{h}$ lies in $\mathfrak{X}(\mathscr{P}) \cap \mathfrak{U}(Q)$. The set $\left\{S^{1}\right\} \cup\left\{S^{1}+(-1)^{h} S^{h}\right\}$ has the same span as $\left\{S^{h} \mid h \geq 1\right\}$, and each element except (possibly) $S^{1}$ of the new set is in $\mathfrak{Y}(\mathscr{P}) \cap \mathfrak{U}(Q) \subset \mathfrak{U}(\mathscr{P})+\mathfrak{U}(Q)$. Since the basis $p$-relations not of the form $S^{h}$ are in $\mathfrak{U}(\mathscr{P})+\mathfrak{U}(Q)$ [in fact, they are in $\mathfrak{Y}(\mathscr{P}) \cup \mathfrak{Y}(Q)], \mathfrak{U}(\mathscr{P})+\mathfrak{Y}(Q)$ has codimension $\leq 1$ in $\mathscr{S}$. q.e.d.

We shall now see that this codimension is exactly one.

Let $T^{\prime} \in \mathscr{R}$ be the curvature operator which interchanges $\mathscr{P}$ and $Q$ and is zero on $\mathscr{L}\{\mathscr{P}, Q\}^{\perp}$. Let $T$ be the projection of $T^{\prime}$ onto $\mathscr{S}$.

Lemma C. $T$ spans $[\mathfrak{U}(\mathscr{P})+\mathfrak{Y}(Q)]^{\perp}$.

Proof of Lemma C. We shall make repeated use of the following fact: for all $S \in \mathscr{S}$, 


$$
\langle T, S\rangle=\left\langle T^{\prime}, S\right\rangle=2\langle S(\mathscr{P}), Q\rangle /\left(1-\langle\mathscr{P}, Q\rangle^{2}\right) \text {. }
$$

To see this, compute $\operatorname{tr}\left(S \circ T^{\prime}\right)$ using an orthonormal basis for $\Lambda^{p}$ containing $\mathscr{P}$ and $(Q-\langle Q, \mathscr{P}\rangle \mathscr{P}) /\left(1-\langle Q, \mathscr{P}\rangle^{2}\right)^{1 / 2}$.

It is now clear that $T$ is orthogonal to $\mathfrak{U}(\mathscr{P})$ and $\mathscr{U}(Q)$. If $T^{\prime}$ does not satisfy the Bianchi identities, then $T \neq 0$ and $T$ spans $[\mathfrak{U}(\mathscr{P})+\mathscr{H}(Q)]^{\perp}$. But $\mathscr{P}+Q$ is not decomposable, and so there exists $S_{0} \in \mathscr{S}$ such that $\beta=\left\langle S_{0}(\mathscr{P}+Q), \mathscr{P}+Q\right\rangle$ $\neq 0$. Since both $\mathscr{P}$ and $Q$ are decomposable, $\beta=2\left\langle S_{0}(\mathscr{P}), Q\right\rangle$. Then $\left\langle T^{\prime}, S_{0}\right\rangle$ $=\beta /\left(1-\langle\mathscr{P}, Q\rangle^{2}\right) \neq 0$ and $T^{\prime} \notin \mathscr{B}$. Therefore $\mathscr{S}$ is spanned by $\mathfrak{U}(\mathscr{P}), \mathfrak{U}(Q)$, and $T$. q.e.d.

It follows that $S_{g}$ and $S_{Q}$ may be written

$$
S_{\mathscr{q}}=S_{\mathscr{g}}^{\mathscr{g}}+S_{\mathscr{q}}^{Q}+\left\langle S_{\mathscr{g}}, T\right\rangle T /\|T\|^{2}, \quad S_{Q}=S_{Q}^{\mathscr{s}}+S_{Q}^{Q}+\left\langle S_{Q}, T\right\rangle T /\|T\|^{2},
$$

where $S_{\mathscr{g}}^{\mathscr{P}}, S_{Q}^{\mathscr{P}} \in \mathfrak{U}(\mathscr{P})$ and $S_{\mathscr{q}}^{Q}, S_{Q}^{Q} \in \mathfrak{U}(Q)$. But since $S_{\mathscr{q}}, S_{Q} \in \mathscr{S}$,

$$
\left\langle S_{\mathscr{P}}, T\right\rangle=\frac{2\left\langle S_{\mathscr{P}}(\mathscr{P}), Q\right\rangle}{1-\langle\mathscr{P}, Q\rangle^{2}}=\frac{2\langle R(\mathscr{P}), Q\rangle}{1-\langle\mathscr{P}, Q\rangle^{2}}=\frac{2\langle R(Q), \mathscr{P}\rangle}{1-\langle\mathscr{P}, Q\rangle^{2}}=\left\langle S_{Q}, T\right\rangle .
$$

Denoting $\left\langle S_{\mathscr{g}}, T\right\rangle /\|T\|^{2}=\left\langle S_{Q}, T\right\rangle /\|T\|^{2}$ by $\alpha$, we have $S=S_{\mathscr{g}}^{Q}+S_{Q}^{\mathscr{F}}+\alpha T$ satisfies $\{\mathscr{P}, Q\} \subset \operatorname{Ker}(R-S)$ as desired. Theorem 3.6 is proved for the case $\mathscr{P}+Q$ not decomposable.

Case 2: $\mathscr{P}+Q$ decomposable. In this case, $\mathscr{P}_{t}=[1+2(\cos t)(\sin t)$ $\langle\mathscr{P}, Q\rangle]^{-1 / 2}[(\cos t) \mathscr{P}+(\sin t) Q]$ is decomposable for all values of $t$ by Lemma A. In fact, $\mathscr{P}_{t}$ is in $Z(R)$, for

$$
\sigma_{R}\left(\mathscr{P}_{t}\right)=\frac{2(\cos t) \sin t}{1+2(\cos t)(\sin t)\langle\mathscr{P}, Q\rangle}\langle R(\mathscr{P}), Q\rangle .
$$

Since $\sigma_{R} \geq 0$ and the coefficient of $\langle R(\mathscr{P}), Q\rangle$ above changes sign, $\langle R(\mathscr{P}), Q\rangle$ $=0$ and $\sigma_{R}\left(\mathscr{P}_{t}\right)=0$ for all $t$. Thus we see that $\mathscr{P}$ and $Q$ determine a circle in $\mathscr{G}$ of zeros (and therefore critical zeros) of $\sigma_{R}$. For any $S \in \mathscr{S}, \mathscr{P}$ and $Q$ are in $\operatorname{Ker}(R-S)$ if and only if $\mathscr{P}_{t}$ is in $\operatorname{Ker}(R-S)$ for all $t$, so we may assume $\mathscr{P} \perp Q$.

We choose an orthonormal basis for $V$ so that $\mathscr{P}=e_{1} \wedge \cdots \wedge e_{p}$ and $Q=e_{1} \wedge \cdots \wedge e_{p-1} \wedge e_{p+1}$. By Proposition 3.2, there exist $\mu_{i J}$ and $\nu_{k J}$ in $\boldsymbol{R}$ so that $S_{\mathscr{g}}=\sum \mu_{i J} S_{I J}$ and $S_{Q}=\sum \nu_{k J} S_{K J}$ satisfy $\mathscr{P} \in \operatorname{Ker}\left(R-S_{\mathscr{g}}\right)$ and $Q \in \operatorname{Ker}\left(R-S_{Q}\right)$. The sum in the expression for $S_{g}$ ranges over all $I, J$ such that $S_{I J}$ is in the basis for $\mathscr{S}$ and $I=\{1, \cdots, p, i\}$, and for $S_{Q}$ ranges over all $K, J$ such that $S_{K J}$ is in the basis and $K=\{1, \cdots, p-1, p+1, k\}$. Note that for such $I$ and $K$,

$$
\begin{aligned}
& S_{I J}(\mathscr{P})=(-1)^{p+1} e_{i} \wedge e_{j_{1}} \wedge \cdots \wedge e_{j_{p-1}}, \\
& S_{K J}(Q)=(-1)^{p+1} e_{k} \wedge e_{j_{1}} \wedge \cdots \wedge e_{j_{p-1}} .
\end{aligned}
$$


We ask again which of the basis $p$-relations lie in $\mathfrak{Y}(\mathscr{P})+\mathfrak{X}(Q)$.

Lemma D. If $\mathscr{P}=e_{1} \wedge \cdots \wedge e_{p}$ and $Q=e_{1} \wedge \cdots \wedge e_{p-1} \wedge e_{p+1}$, then $\mathfrak{H}(\mathscr{P})+\mathfrak{A}(Q)$ contains all of the basis p-relations except possibly $S^{\lambda, i}=$ $S_{1 \cdots(p+1) 1 \cdots \hat{\lambda} \cdot \cdot(p-1) i}$ for $i>p+1$ and $\lambda \leq p-1$.

Proof of Lemma D. We shall show that most of the basis $p$-relations are in either $\mathfrak{A}(\mathscr{P})+\mathfrak{U}(Q)$ and that, except for those listed above, the rest are expressible as $A+B$ where $A \in \mathfrak{U}(\mathscr{P})$ and $B \in \mathfrak{X}(Q)$.

An argument similar to that used for in Lemma B shows that the only basis $p$-relations which annihilate neither $\mathscr{P}$ nor $Q$ are $S_{1 \cdots p+1 J}$ where $J=\left\{j_{1}, \cdots, j_{p-1}\right\}$ $\not \subset\{1, \cdots, p+1\}$, i.e., $j_{p-1}>p+1$.

If $\left\{j_{1}, \cdots, j_{p-2}\right\} \neq\{1, \cdots, \hat{\lambda}, \cdots, p-1\}$ for some $\lambda \leq p-1$, then write

$$
S_{1 \cdots p+1 J}=\left(S_{1 \cdots p+1 J}+S_{1 \cdots p j_{p-1} j_{1} \cdots j_{p-2} p+1}\right)-S_{1 \cdots p j_{p-1} j_{1} \cdots j_{p-2} p+1} .
$$

But the summand in parentheses is in $\mathscr{A}(\mathscr{P})$, and the other summand is in $\mathfrak{U}(Q)$. Therefore $S_{1 \ldots p+1 J}$ is in $\mathfrak{Y}(\mathscr{P})+\mathfrak{U}(Q)$ in this case. The only basis $p$-relations which may not be in $\mathfrak{U}(\mathscr{P})+\mathfrak{U}(Q)$ are $S_{1 \ldots p+1 J}$ such that

$$
\left\{j_{1}, \cdots, j_{p-2}\right\}=\{1, \cdots, \hat{\lambda}, \cdots, p-1\} \quad \text { and } j_{p-1}>p+1 . \quad \text { q.e.d. }
$$

We can now write the expressions for $S_{\mathscr{g}}$ and $S_{Q}$ as

$$
S_{\mathscr{g}}=S_{\mathscr{g}}^{\mathscr{P}}+S_{\mathscr{g}}^{Q}+\sum_{\substack{\lambda \leq p-1 \\ i>p+1}} \alpha_{\lambda, i} S^{\lambda, i}, \quad S_{Q}=S_{Q}^{\mathscr{P}}+S_{Q}^{Q}+\sum_{\substack{\lambda \leq p-1 \\ i>p+1}} \beta_{\lambda, i} S^{\lambda, i},
$$

where $S_{\mathscr{g}}^{\mathscr{g}}, S_{Q}^{\mathscr{p}} \in \mathfrak{A}(\mathscr{P})$ and $S_{\mathscr{g}}^{Q}, S_{Q}^{Q} \in \mathfrak{U}(Q)$. If we establish that $\alpha_{\lambda, i}=\beta_{\lambda, i}$, then

$$
S=S_{\mathscr{F}}^{Q}+S_{Q}^{\mathscr{P}}+\sum \alpha_{\lambda, i} S^{\lambda, i}
$$

satisfies $\{\mathscr{P}, Q\} \subset \operatorname{Ker}(R-S)$.

To show that $\alpha_{\lambda, i}=\beta_{\lambda, i}$ let $E^{\lambda, i}=e_{1} \wedge \cdots \wedge \hat{e}_{\lambda} \wedge \cdots \wedge e_{p-1} \wedge e_{p+1} \wedge e_{i}$. By (9), $\alpha_{\lambda, i}=(-1)^{p}\left\langle S_{\mathscr{g}}(\mathscr{P}), E^{\lambda, i}\right\rangle$. Now since $E^{\lambda, i}+Q$ is decomposable, $0=\left\langle S\left(E^{\lambda, i}+Q\right), E^{\lambda, i}+Q\right\rangle=2\left\langle S(Q), E^{\lambda, i}\right\rangle$ for all $S \in \mathscr{S}$. In particular, $\left\langle S_{Q}(Q), E^{\lambda, i}\right\rangle=0$. Therefore we have

$$
\begin{aligned}
\alpha_{\lambda, i} & =(-1)^{p}\left\langle S_{\mathscr{P}}(\mathscr{P}), E^{\lambda, i}\right\rangle=(-1)^{p}\left\langle S_{\mathscr{P}}(\mathscr{P})+S_{Q}(Q), E^{\lambda, i}\right\rangle \\
& =\sqrt{2}(-1)^{p}\left\langle R[(\mathscr{P}+Q) / \sqrt{2}], E^{\lambda, i}\right\rangle .
\end{aligned}
$$

Since $(\mathscr{P}+Q) / \sqrt{2}$ is a critical zero of $\sigma_{R}$, there exists $\tilde{S} \in \mathscr{S}$ so that $R[(\mathscr{P}+Q) / \sqrt{2}]=\tilde{S}[(\mathscr{P}+Q) / \sqrt{2}]$.

Writing $\tilde{S}$ in terms of the basis $p$-relations, $\tilde{S}=\sum \gamma_{I J} S_{I J}$. We now have

$$
\begin{aligned}
\alpha_{\lambda, i} & =(-1)^{p}\left\langle\tilde{S}(\mathscr{P}+Q), E^{\lambda, i}\right\rangle \\
& =(-1)^{p} \sum \gamma_{I J}\left[\left\langle S_{I J}(\mathscr{P}), E^{\lambda, i}\right\rangle+\left\langle S_{I J}(Q), E^{\lambda, i}\right\rangle\right] \\
& =(-1)^{p} \sum \gamma_{I J}\left\langle S_{I J}(\mathscr{P}), E^{\lambda, i}\right\rangle
\end{aligned}
$$


since $\left\langle S_{I J}(Q), E^{\lambda, i}\right\rangle=0$. But applying (4), $S_{I J}(\mathscr{P})$ is either zero or orthogonal to $E^{\lambda, i}$ except for $S^{\lambda, i}$. Recall that $\left\langle S^{\lambda, i}(\mathscr{P}), E^{\lambda, i}\right\rangle=(-1)^{p}$. Therefore $\alpha_{\lambda, i}=$ $\gamma_{1} \cdots(p+1) 1 \cdots \hat{k} \cdots(p-1) i$.

Similarly, letting $F^{\lambda, i}=e_{1} \wedge \cdots \wedge \hat{e}_{\lambda} \wedge \cdots \wedge e_{p-1} \wedge e_{p} \wedge e_{i}, \mathscr{P}+F^{\lambda, i}$ is decomposable and

$$
\begin{aligned}
\beta_{\lambda, i} & =(-1)^{p-1}\left\langle S_{Q}(Q), F^{\lambda, i}\right\rangle=(-1)^{p-1}\left\langle\tilde{S}(\mathscr{P}+Q), F^{\lambda, i}\right\rangle \\
& =(-1)^{p-1} \sum \gamma_{I J}\left\langle S_{I J}(Q), F^{\lambda, i}\right\rangle \\
& =(-1)^{p-1} \gamma_{1 \cdots(p+1) 1 \cdots \hat{\lambda} \cdots(p-1) i}\left\langle S^{\lambda, i}(Q), F^{\lambda, i}\right\rangle \\
& =\gamma_{1 \cdots(p+1) 1 \cdots \hat{\lambda} \cdots(p-1) i}=\alpha_{\lambda, i} .
\end{aligned}
$$

This establishes Theorem 3.6 for $\mathscr{P}+Q$ decomposable. q.e.d.

We continue to piece together the $S$ 's which work for individual zeros of $\sigma_{R}$ into one which works for them simultaneously.

Lemma 3.7. If $Z$ is any subset of $\mathscr{G}$, there exists a finite subset $\left\{\mathscr{P}_{1}, \cdots, \mathscr{P}_{k}\right\} \subset Z$ such that $\mathfrak{A}(Z)=\mathfrak{A}\left\{\mathscr{P}_{1}, \cdots, \mathscr{P}_{k}\right\}$.

Proof. Let $\mathscr{P}_{1}, \cdots, \mathscr{P}_{k}$ be planes in $Z$ which span $\mathscr{L}\{\mathscr{P} \mid \mathscr{P} \in Z\}$.

Theorem 3.8. Let $R \in \mathscr{R}$ have nonnegative sectional curvature. Then there exists $S \in \mathscr{S}$ with

$$
\left\{\mathscr{P} \in \mathscr{G} \mid \sigma_{R}(\mathscr{P})=0\right\}=\mathscr{G} \cap \operatorname{Ker}(R-S) .
$$

Proof. We construct the unique $S \in \mathfrak{A}(Z(R))^{\perp}$ which has the desired property. Let $\mathscr{P}_{1}, \cdots, \mathscr{P}_{k}$ be as in Lemma 4.7 where $Z=\left\{\mathscr{P} \in \mathscr{G} \mid \sigma_{R}(\mathscr{P})=0\right\}$. Then

$$
\mathfrak{U}(Z)^{\perp}=\sum_{i=1}^{k} \mathfrak{U}\left(\mathscr{P}_{i}\right)^{\perp}
$$

Let $\mathrm{S}_{i} \in \mathfrak{X}\left(\mathscr{P}_{i}\right)^{\perp}$ be such that $\mathscr{P}_{i} \in \operatorname{Ker}\left(R-S_{i}\right)$. By Theorem 3.6, for each $i, j$ there exists a unique $S_{i j} \in \mathfrak{Y}\left(\mathscr{P}_{i}\right)^{\perp}+\mathfrak{U}\left(\mathscr{P}_{j}\right)^{\perp}$ such that $\left\{\mathscr{P}_{i}, \mathscr{P}_{j}\right\} \subset$ $\operatorname{Ker}\left(R-S_{i j}\right)$. By Lemma 3.3, $S_{i}$ is the orthogonal projection in $\mathscr{U}(Z)^{\perp}$ of $S_{i j}$ onto $\mathfrak{U}\left(\mathscr{P}_{i}\right)^{\perp}$. Lemma 5.3 of [5] now guarantees a unique $S$ in $\mathfrak{U}(Z)^{\perp}$ whose projection onto $\mathfrak{X}\left(\mathscr{P}_{i}\right)^{\perp}$ is $S_{i}$. Using Lemma 3.3 again, $\mathscr{P}_{i} \in \operatorname{Ker}(R-S)$ for all $i \leq k$, and so $Z \subset \operatorname{Ker}(R-S)$. Finally, if $\mathscr{P} \in \mathscr{G} \cap \operatorname{Ker}(R-S)$, $\sigma_{R}(\mathscr{P})=\sigma_{S}(\mathscr{P})=0$.

Corollary 3.9. If $R \in \mathscr{R}$, and $\lambda$ is the minimum (respectively, maximum) value of $\sigma_{R}$ on $\mathscr{G}$, then there exists $S \in \mathscr{S}$ such that the set of p-planes on which $\sigma_{R}$ assumes the value $\lambda$ is $\mathscr{G} \cap \operatorname{Ker}(R-\lambda I-S)$.

Proof. Immediate from Theorem 3.8 since $\sigma_{R-\lambda I} \geq 0$ (respectively, $\sigma_{\lambda I-R} \geq 0$ ). q.e.d.

In the proof of Theorem 3.8, the fact that zero is the minimum of $\sigma_{R}$ is used only to show that if $\mathscr{P}+Q$ is decomposable, the zero $\mathscr{P}_{t}$ is a critical point of $\sigma_{R}$ (last part of Theorem 3.6). The theorem cannot be weakened 
however to the case of zero an arbitrary critical value of $\sigma_{R}$, as the following example shows.

Let $A$ be the linear transformation on $V$ which interchanges $e_{i}$ and $e_{i+p}$ if $i \leq p$, and is zero on $e_{j}$ if $j>2 p$. Then $A$ is self-adjoint and so induces a self-adjoint transformation $R$ on $\Lambda^{p}(V)$. Each of the basis planes $E_{i}=$ $e_{i_{1}} \wedge \cdots \wedge e_{i_{p}}$ of $\Lambda^{p}$ is a critical zero of $\sigma_{R}$ :

$$
\operatorname{grad} \sigma_{R}\left(E_{i}\right)=2 R\left(E_{i}\right)=2\left(A e_{i_{1}} \wedge \cdots \wedge A e_{i_{p}}\right)=0 \text { or } \pm 2 * E_{i},
$$

which is normal to $\mathscr{G}$ at $E_{i}$ by Lemma 3.1. Here $*$ is the star operator on $\Lambda^{p}\left(\mathscr{L}\left\{e_{1}, \cdots, e_{2 p}\right\}\right)$. If the theorem were true for zero an arbitrary critical value of $\sigma_{R}, Z(R)$ would have to be all of $\mathscr{G}$. But

$$
Q=\left(e_{1} \pm e_{p+1}\right) \wedge\left(e_{2} \pm e_{p+2}\right) \wedge \cdots \wedge\left(e_{p} \pm e_{2 p}\right) /(\sqrt{2})^{p}
$$

is in $\mathscr{G}$ and $R(Q)=( \pm)^{p} Q$, so $\sigma_{R}(Q)=( \pm 1)^{p} \neq 0$. $\sigma_{R}$ takes on values of either sign. It is shown in [3] that curvature operators such as this one, induced by transformations of $V$, satisfy the Bianchi identities.

On the other hand we may conclude: if $\lambda$ is a critical value of $\sigma_{R}$, and $\left\{\mathscr{P} \in \mathscr{G} \mid \sigma_{R}(\mathscr{P})=\lambda\right\}$ contains no pairs of planes which intersect in a $(p-1)$ dimensional subspace, then there exists $S \in \mathscr{S}$ for which

$$
\left\{\mathscr{P} \in \mathscr{G} \mid \sigma_{R}(\mathscr{P})=\lambda\right\}=\mathscr{G} \cap \operatorname{Ker}(R-\lambda I-S)
$$

\section{References}

[ 1] W. V. D. Hodge \& D. Pedoe, Methods of algebraic geometry, Cambridge University Press, Cambridge, 1947.

[2] R. S. Kulkarni, Curvature structures in differential geometry, to appear.

[ 3 ] A. K. Stehney, The Grassmann quadratic p-relations and curvature, Thesis, State University of New York at Stony Brook, 1971.

[4] J. A. Thorpe, Some remarks on the Gauss-Bonnet integral, J. Math. Mech. 18 (1969) 779-786.

[5] - The Zeroes of nonnegative curvature operators, J. Differential Geometry 5 (1971) 113-126.

[6] _- On the curvature tensor of a positively curved 4-manifold, Proc. 13th Biennial Sem. Canad. Math. Congress, 1971,.156-159.

Wellesley College 